\title{
Jet Studies in STAR via Di-jet Triggered (2+1) Multi-hadron Correlations
}

\author{
Kolja Kauder for the STAR Collaboration \\ University of Illinois at Chicago, Chicago, IL 60607, USA
}

\begin{abstract}
We explore jet-medium interactions via the recently developed multi-hadron correlation or " $2+1$ " technique. We restrict the di-jet kinematics by selecting a pair of approximately back-to-back high $p_{T}$ hadron triggers and study associated particles. Here we present our study of di-jet systematics comparing the measurements of associate yields and spectra in $200 \mathrm{GeVAu}+\mathrm{Au}$ and $\mathrm{d}+\mathrm{Au}$ collisions in two different scenarios. We present $\sum p_{T}$ as an estimate for energy loss. First findings indicate little to no energy loss in the symmetric " $2+1$ " scenario whereas model predictions are about $2 \mathrm{GeV}$.
\end{abstract}

\section{Introduction}

A strongly interacting partonic medium is evident in multiple observables at RHIC. One manifestation of this medium, the jet-quenching effect, was found in single-particle spectra [1] as well as in away-side di-hadron angular correlations [2]. The latter have already yielded new insights into the medium properties: away-side quenching indicates that the medium is dense, and transverse momentum distributions of associated hadrons are consistent with thermalization [2]. Alteration of the angular distribution on both (same- and away-) sides of a high $p_{T}$ trigger reveals a strong modification of known hadron production mechanisms. Alternatively, it has been argued that the peculiar features found in the triggered di-hadron distributions, such as a doublehumped away-side structure [3] and a "ridge" along pseudo-rapidity $\Delta \eta$ formed on the same-side of the trigger, could be caused by the medium's response to propagated jets [4].

Several three-particle correlation techniques have been developed to further investigate proposed mechanisms for jet modifications like jet deflection or the Mach cone/shock wave mechanism. Examples include $\Delta \phi-\Delta \phi$ correlation analyses [5] and $\Delta \eta-\Delta \eta$ studies [6]. Both methods use one high $p_{T}$ trigger particle and then study the correlation of two lower $p_{T}$ associate particles, and in that sense are denoted as $1+2$ techniques. With our $2+1$ method we instead use a pair of correlated high $p_{T}$ hadron triggers to determine the jet axis and study associated hadron distributions with respect to this axis. Thus we aim to explore both same- and away-side features with a well-defined jet axis at the same time. This new approach has already shown unique results [7]; here we present the most recent developments.

\section{Data Analysis}

A description of the method and initial results were introduced in [7]. To summarize, the re-emergence of an away-side peak in angular correlations of two high $p_{T}$ particles [8] allows Preprint submitted to Nuclear Physics A

August 14, 2019 
us to determine the away-side jet direction in both azimuth and pseudo-rapidity. The highest transverse momentum particle in the event is designated as a primary trigger (T1) if it is at midrapidity, $|\eta|<1$, and if it satisfies the transverse momentum threshold. A second trigger (T2) is selected back-to-back with the primary trigger, i.e. within $|\Delta \phi-\pi|<\alpha$, where $\Delta \phi$ is the relative azimuthal angle between the two triggers. We choose $\alpha=0.2$ as a characteristic away-side peak width for this kinematic regime. T2 satisfies the same pseudo-rapidity cuts and both triggers have to obey additional track quality cuts. The distribution of associated hadrons $(\mathrm{A})$ is then studied in both $\Delta \eta$ and $\Delta \phi$ dimensions with respect to the jet axis as determined by this pair of triggers. The associated hadrons have to satisfy similar track quality cuts as the trigger pair.

The raw angular correlation is constructed similarly to other di-hadron analyses [3, 4]. To correct for pair acceptance we use the mixed-event technique. The combinatorial background is modulated by the $\cos (2 \Delta \phi)$ function to account for elliptic flow effects. We use published STAR results for the centrality and $p_{T}$ dependence of the elliptic flow coefficient $v_{2}$ [9]. The overall background level is determined via the Zero-Yield at Minimum (ZYAM) method as in [10].

Specific to $2+1$ correlations is the correlated background, resulting from randomly associated trigger pairs. The angular distribution of the associated hadrons for these trigger pairs is obtained from di-hadron correlations with appropriate kinematic selections. We scale this background by the signal-to-noise ratio in the away-side peak trigger-trigger correlation used for jet axis determination and subtract this contribution from the raw distribution. The resulting corrected signal is scaled back so that ultimately the correlation is normalized per di-jet trigger.

\section{Results}
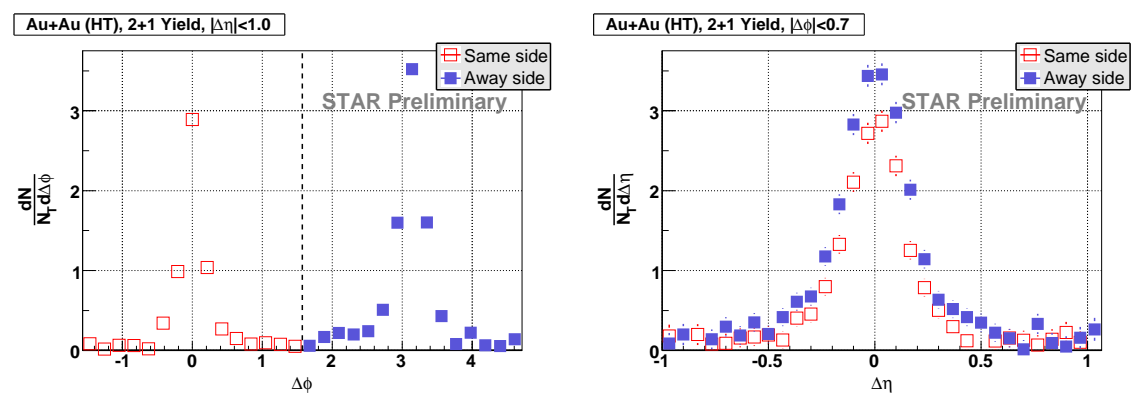

Figure 1: Yields in the asymmetric case (normalized per number of triggers $\mathrm{N}_{T}$ ): High-Tower triggered $200 \mathrm{GeV} \mathrm{Au+Au}$ data, $8 \mathrm{GeV}<E_{T}(T 1)<15 \mathrm{GeV}, 4 \mathrm{GeV} / c<p_{T}(T 2)<\min \left\{p_{T}(T 1), 10 \mathrm{GeV} / c\right\}, 1.5 \mathrm{GeV} / c<p_{T}(A)<10 \mathrm{GeV} / c$. Upward triangles signify the same-side correlation and are compared directly with the away-side correlation in the downward triangles. $\Delta \phi$ and $\Delta \eta$ are calculated with respect to T1 on the same-side and with respect to T2 on the awayside. The left figure shows the $\Delta \phi$ projection; the right figure shows the $\Delta \eta$ projection. Error bars are statistical only.

Below we consider two scenarios with different relative $p_{T}$ between the two trigger particles. The first is a symmetric scenario, as in our early results [7], in which the T1 trigger is selected between 5 and $10 \mathrm{GeV} / c$ and $\mathrm{T} 2$ above $4 \mathrm{GeV} / c$. Associated hadrons were selected with $p_{T}$ between 1.5 and $4 \mathrm{GeV} / c$. 20M $200 \mathrm{GeV}$ Central Au+Au events were used for this analysis. The availability of the High-Tower triggered $200 \mathrm{GeV} \mathrm{Au+Au}$ data set in STAR with good statistics in the higher $p_{T}$ region enabled us to consider a second, asymmetric scenario. Using the Barrel Electromagnetic Calorimeter, we required the T1 trigger's transverse energy $E_{T}$ to be 
between 8 and $15 \mathrm{GeV}$, rejecting triggers that coincided with a charged hadron track. The selection criteria for $\mathrm{T} 2$ remained unchanged, associated particles were selected to have transverse momenta in the TPC above $1.5 \mathrm{GeV} / c$. Where $p_{T}$ cuts were used, values above the resolution threshold of $10 \mathrm{GeV} / c$ disqualified an event in both scenarios.

Previously, we found no modifications on the away-side of a di-jet in the symmetric case [7]. In the present analysis we confirmed the absence of the jet-medium modification effects for such di-jets with more detailed studies of transverse momentum spectra of associated hadrons. A tangential jet production scenario could account for such results.

In contrast, an asymmetric trigger pair should enhance long path lengths through the medium on the away-side. The correlation structures resulting from a $2+1$ analysis for the asymmetric triggers feature clear jet-like peaks on both same- and away-sides. $\Delta \phi$ and $\Delta \eta$ projections of the 2-dimensional correlation function are shown in Figure 1, with no obvious broadening or indication of a ridge-like phenomenon. In this new analysis we observe enhancement rather than suppression of away-side yield in contrast to all di-hadron correlation results for this kinematic range of associated particles. We next investigate the correlation peaks in more detail.
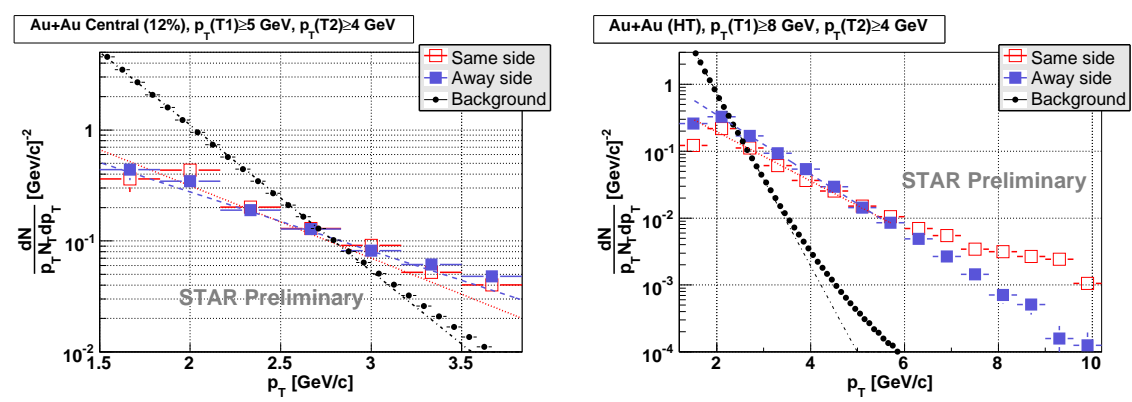

Figure 2: $\quad p_{T}$ spectra (normalized per number of triggers $\mathrm{N}_{T}$ ) for the symmetric case on the left and the asymmetric case on the right. Upward triangles signify the same-side correlation while the away-side correlation is represented by downward triangles. In both cases the minimum bias inclusive background is given as a reference. As a visual aid fits to $e^{x} / x$ are shown with dashed lines. Error bars are statistical only.

Figure 2 shows the $p_{T}$ spectra of associated hadrons in the jet peaks as defined by $|\Delta \eta|<0.5$ and $|\Delta \phi|<0.5$ for both cases. Note the expected significant hardening of the associated hadron spectra on both sides of the primary trigger as compared to the inclusive hadron distribution. Also, just as in the case of angular variables, the spectra on both sides are virtually identical for the symmetric case. On the other hand, the away-side spectrum of the asymmetric trigger pair is softer than that of the same-side. Comparison of symmetric di-jet spectra between Central $\mathrm{Au}+\mathrm{Au}$ and minimum bias $\mathrm{d}+\mathrm{Au}$ data (available from Run III) confirmed the absence of modifications within our uncertainties. Analysis of the $d+A u$ data from Run VIII is in progress.

To further quantify our findings the average $p_{T}$ of associated hadrons and the total yield in the jet peaks for both scenarios are shown in Figure 3 We are finalizing the systematic uncertainties, but they are expected to be between $20 \%$ and $30 \%$ and strongly correlated between same- and away-side. If this expectation holds, there will be no appreciable difference between $\mathrm{d}+\mathrm{Au}$ and $\mathrm{Au}+\mathrm{Au}$ systems in the symmetric case. By contrast, in the asymmetric case, we see a spread between same- and away-side. The enhancement in total yield is offset by an attenuation in $\left\langle p_{T}\right\rangle$.

In [11] two models were used to compute total energy deposition in the medium for di-hadron triggered events. As a proxy for this value we sum up the $p_{T}$ of trigger and associated particles on 

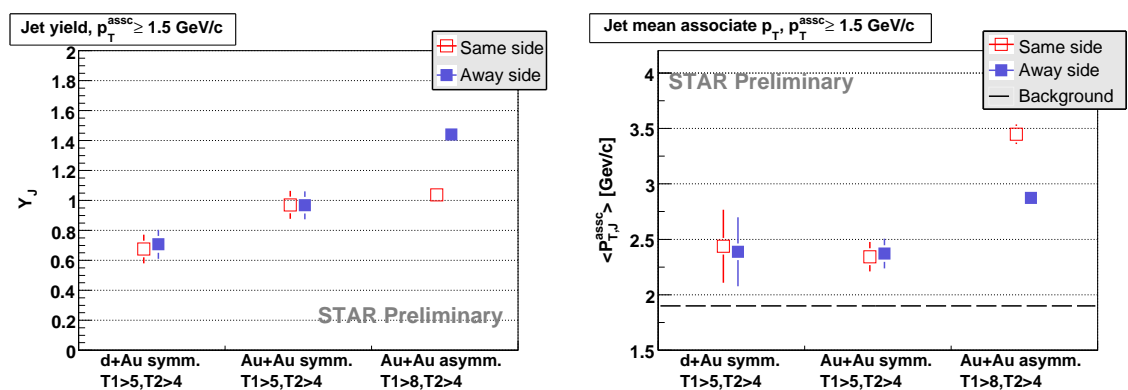

Figure 3: Yield (left) and $\left\langle p_{T}\right\rangle$ (right) in the symmetric and asymmetric case. For the symmetric case, the d+Au values are also shown. Upward triangles are used for the same-side correlation, downward triangles for the away-side correlation. Error bars are statistical only.

both same- and away-sides and consider the difference between the two. In the symmetric case we find similar values of $1.50 \pm 0.31 \mathrm{GeV} / c(\mathrm{Au}+\mathrm{Au})$ and $1.64 \pm 0.35 \mathrm{GeV} / c(\mathrm{~d}+\mathrm{Au})$, consistent with the estimate for $k_{T}$ smearing used in [11]. The differences between the correlation peaks, if any, are not accessible with these measurements. For the asymmetric case we do not yet have a comparison with $\mathrm{d}+\mathrm{Au}$. A reasonable assumption is that the $k_{T}$ effect has about the same trigger-independent value of $1.6 \mathrm{GeV} / c$. Subtracting this from the $\mathrm{Au}+\mathrm{Au} \sum p_{T}$ difference of $4.3 \mathrm{GeV} / c$ results in an estimated energy loss of $3 \mathrm{GeV}$ in the fiducial range.

\section{Summary}

In correlations using symmetric $p_{T}$ trigger pairs we found no evidence for modifications in yield or $p_{T}$ spectra for both the Central $\mathrm{Au}+\mathrm{Au}$ and $\mathrm{d}+\mathrm{Au}$ collisions, and for same- and awaysides. This is consistent with minimal medium interaction, e.g. strong surface bias in the $\mathrm{Au}+\mathrm{Au}$ correlation. We expanded our $2+1$ correlation studies of di-jet properties to include an asymmetric trigger selection scenario and again found the peaks on the same-side and away-side to be similar in shape. However, the away-side exhibited an increased yield balanced by a softer spectrum resulting in a lower $\left\langle p_{T}\right\rangle$.

We presented $\sum p_{T}$ as an estimate for energy loss, and our first results suggest negligible energy loss in the symmetric region where model predictions are about $2 \mathrm{GeV}$. We are working to complete $d+A u$ reference calculations for the asymmetric case. Our initial findings indicate energy loss values that are at the lower end of predictions.

\section{References}

[1] S. S. Adler et al., Phys. Rev. Lett. 91 (2003) 072303

[2] J. Adams et al., Phys. Rev. Lett. 95 (2005) 152301.

[3] M. J. Horner, J. Phys. G 34 (2007) S995.

[4] J. Putschke, J. Phys. G 34 (2007) S679.

[5] J. G. Ulery, Nucl. Phys. A783 (2007) 511-514.

[6] B. I. Abelev, Phys. Rev. Lett.102 (2009) 052302.

[7] O. Barannikova, J. Phys. G35 (2008) 104086.

[8] J. Adams et al., Phys. Rev. Lett. 97 (2006) 162301.

[9] J. Adams et al., Phys. Rev. C 72 (2005) 014904.

[10] J. G. Ulery and F. Wang, Nucl. Instrum. Meth. A 595 (2008) 502.

[11] T. Renk, Phys. Rev. C 78(1) (2008) 014903. 